\title{
Baicalein suppresses 17- $\beta$-estradiol-induced migration, adhesion and invasion of breast cancer cells via the $G$ protein-coupled receptor 30 signaling pathway
}

\author{
DANDAN SHANG ${ }^{1}$, ZHENG LI $^{1}$, ZHUXIA ZHU ${ }^{1}$, HUAMEI CHEN ${ }^{1}$, LUJUN ZHAO ${ }^{2,3}$, \\ $\mathrm{XUDONG} \mathrm{WANG}^{1}$ and YAN CHEN ${ }^{1-3}$ \\ ${ }^{1}$ Department of Physiology/Cancer Research Group, Guiyang Medical University School of Basic Medicine; \\ ${ }^{2}$ Department of Pharmacology of Chinese Material Medica and ${ }^{3}$ Key Laboratory of Optimal Utilization \\ of Natural Medicine Resources, Guiyang Medical University, Guiyang, Guizhou 550004, P.R. China
}

Received October 4, 2014; Accepted January 26, 2015

DOI: 10.3892/or.2015.3786

\begin{abstract}
Flavonoids are structurally similar to steroid hormones, particularly estrogens, and therefore have been studied for their potential effects on hormone-dependent cancers. Baicalein is the primary flavonoid derived from the root of Scutellaria baicalensis Georgi. In the present study, we investigated the effects of baicalein on $17 \beta$-estradiol (E2)-induced migration, adhesion and invasion of MCF-7 and SK-BR-3 breast cancer cells. The results demonstrated that baicalein suppressed E2-stimulated wound-healing migration and cell-Matrigel adhesion, and ameliorated E2-promoted invasion across a Matrigel-coated Transwell membrane. Furthermore, baicalein interfered with E2-induced novel G protein-coupled estrogen receptor (GPR30)-related signaling, including a decrease in tyrosine phosphorylation of epidermal growth factor receptor (EGFR) as well as phosphorylation of extracellular signal-regulated kinase (ERK) and serine/threonine kinase Akt, without affecting GPR30 expression. The results also showed that baicalein suppressed the expression of GPR30 target genes, cysteine-rich 61 (CYR61) and connective tissue growth factor $(C T G F)$ induced by E2. Furthermore, baicalein prevented GPR30-related signaling activation and upregulation of $C Y R 61$ and $C T G F$ mRNA levels induced by G1, a specific GPR 30 agonist. The results suggest that baicalein inhibits E2-induced migration, adhesion and
\end{abstract}

Correspondence to: Dr Yan Chen, Department of Pharmacology of Chinese Material Medica, Guiyang Medical University, 9 Beijing Road, Guiyang, Guizhou 550004, P.R. China

E-mail:s0710189@sina.com

Professor Xudong Wang, Department of Physiology, Guiyang Medical University, 9 Beijing Road, Guiyang, Guizhou 550004, P.R. China

E-mail: xdwang@gmc.edu.cn

Key words: baicalein, estrogen, G protein-coupled receptor 30, migration, adhesion, invasion, breast cancer invasion through interfering with GPR30 signaling pathway activation, which indicates that it may act as a therapeutic candidate for the treatment of GPR30-positive breast cancer metastasis.

\section{Introduction}

Breast cancer is the most common malignancy affecting women and is the leading cause of cancer-related mortality among females worldwide (1). Despite advances in the diagnosis and treatment of breast cancer, approximately a third of patients develop metastatic disease, with a median survival time of less than 3 years (2). Preventing metastasis is important for effectively overcoming breast cancer. Estrogen plays an important role, not only in the initiation and proliferation of breast cancer, but also in cancer metastasis (3). Most of the biological effects of estrogen are mediated by binding and activating classic estrogen receptors (ERs), ER $\alpha$ and ER $\beta$, to stimulate the transcription of target genes via genomic and non-genomic mechanisms (4). Based on this knowledge, hormonal therapies that interfere with ER $\alpha$ function are currently applied in patients with ER $\alpha$-positive breast cancer, and beneficial effects have been achieved. Although there is general success with ER $\alpha$ expression and responsiveness to ER $\alpha$-targeted agents, some patients do not respond to endocrine therapy, and most patients appear to have acquired resistance (5). In addition, some types of ER $\alpha$-negative breast cancer cells remain estrogen responsive, indicating the existence of an alternative receptor for estrogen.

$\mathrm{G}$ protein-coupled estrogen receptor (GPR30), as a member of the 7-transmembrane GPR family, mediates both rapid signaling and transcriptional events in response to estrogen. It mediates estrogenic-rapid non-genomic actions, through the $G \beta \gamma$ subunit downstream, release of heparin-bound EGF (HB-EGF) and metalloproteinase (MMP)-dependent transactivation of epidermal growth factor receptors (EGFRs), as well as activation of mitogen-activated protein kinases (MAPKs), adenyl cyclase, and phosphoinositide 3-kinase (PI3K) (6). Subsequently, it also triggers transcriptional responses by activating various transcription factors, such as FOS, JUN, EGRI 
and ATF3 following the protein kinase signaling cascade (7). GPR30 expression is positively associated with the features of breast tumor progression, including tumor size, HER2/neu status, and metastases. Yet, $\mathrm{ER} \alpha$ is inversely associated with HER2/neu and tumor size (8). ER $\alpha$ in breast cancer generally indicates good prognosis and treatment responsiveness with anti-estrogen agents (9); yet, GPR30 is overexpressed in invasive breast cancer and is positively associated with a metastatic phenotype (10). GPR30 is implicated in breast cancer metastasis and it may provide a new potential target for endocrine therapy.

The incidence of breast cancer is much higher in Western countries than in Asian ones. Epidemiological studies contribute these differences to dietary factors, particularly soy consumption. Flavonoids are considered to have a significant potential in breast cancer chemoprevention (11). Their polyphenolic ring is structurally similar to the steroid nucleus of $17 \beta$-estradiol (Fig. 1A) and may have estrogenic or anti-estrogenic activity (12). Baicalein is the primary flavonoid derived from the root of Scutellaria baicalensis Georgi and is also found in soybeans and fruit, which bears the three-ring structure of the flavone backbone with phenolic hydroxyl at the 5', 6', and 7' position (Fig. 1A). It possesses several health benefits including anti-inflammatory, antioxidant, antiviral, neuroprotective, and anticancer effects (13). It has also been shown to have antitumor effects in various hormone-dependent cancers such as breast, colon, and prostate cancer. Previous studies have found that baicalein suppresses $17 \beta$-estradiol (E2)-induced ER transactivation in MCF-7 cells (14) and displaces $>85 \%$ of estradiol binding in mouse uterine cytosol (15). Additionally, baicalein exhibits effective inhibitory activity against E2/insulin-like growth factor 1 (IGF-1)-induced proliferative events in breast cancer cells (16). These investigations propose that baicalein may exert anti-estrogenic activity.

The present study investigated the inhibitory activity of baicalein on E2-induced migration, adhesion, and invasion of MCF-7 and SK-BR-3 breast cancer cells. Furthermore, we investigated its effect on GPR30-related signaling, including phosphorylation of EGFR as well as extracellular signal-regulated kinase (ERK), serine/threonine kinase Akt activation, and GPR30-mediated gene expression.

\section{Materials and methods}

Reagents and antibodies. Baicalein (purity >98\%) was provided by Professor Zhiyu Li (China Pharmaceutical University, Jiangsu, China). It was dissolved in dimethyl sulfoxide (DMSO) as a stock solution at $0.1 \mathrm{M}$ and stored at $-20^{\circ}$ C. E2 and G1 from Sigma-Aldrich (St. Louis, MO, USA) were dissolved in DMSO as a stock solution at $10^{-1} \mathrm{M}$ and stored at $4^{\circ} \mathrm{C}$. Matrigel was obtained from BD Biosciences (Bedford, MA, USA). Bovine serum albumin (BSA) was purchased from Beijing Solarbio Science and Technology Co., Ltd. (Beijing, China). 3-(4,5-Dimethylthiazol-2yl)-2,5-diphenyltetrazolium bromide (MTT) and cytosine $\beta$-D-arabinofuranoside hydrochloride were purchased from Sigma-Aldrich. The GPR30 (N-15)-R antibody (sc-48525-R, polyclonal antibody, rabbit anti-human, 1:800) was purchased from Santa Cruz Biotechnology, Inc. (Santa Cruz, CA, USA).
Primary antibodies against p-ERK (T202/Y204) (AP0484, polyclonal antibody, rabbit anti-human, 1:1,000), extracellular regulated protein kinase 1/2 (ERK1/2) (L352) (BS1112, polyclonal antibody, rabbit anti-human, 1:1,000), and GAPDH (1A6) (MB001, monoclonal antibody, mouse anti-human, 1:1,000) were purchased from Bio-World (Dublin, OH, USA). Primary antibodies for p-Akt (Ser473) (193H12) (\#4058, monoclonal antibody, rabbit anti-human, 1:1,000), Akt (11E7) (\#4685, monoclonal antibody, rabbit anti-human, 1:1,000), p-EGFR (Tyr1173) (53A5) (\#4407, monoclonal antibody, rabbit anti-human, 1:1,000) and EGFR (D38B1) (\#4267, monoclonal antibody, rabbit anti-human, 1:1,000) were purchased from Cell Signaling Technology (Beverly, MA, USA). The anti-mouse or anti-rabbit IgG horseradish peroxidase-conjugated secondary antibodies $(1: 3,000)$ were purchased from Santa Cruz Biotechnology, Inc.

Cell lines and culture. Human breast cancer cell lines MCF-7 and SK-BR-3 were obtained from the Kunming Cell Bank of the Chinese Academy of Sciences (Yunnan, China). MCF-7 cells were maintained in Dulbecco's modified Eagle's medium (DMEM) with 10\% FBS, and SK-BR-3 cells were maintained in RPMI-1640 (all from Hyclone, Logan, UT, USA) with $10 \%$ FBS (Gibco-BRL, Carlsbad, CA, USA), both supplemented with $100 \mathrm{U} / \mathrm{ml}$ penicillin and $100 \mu \mathrm{g} / \mathrm{ml}$ streptomycin (both from Sigma-Aldrich). These cells were incubated at $37^{\circ} \mathrm{C}$ in humidified air with $5 \% \mathrm{CO}_{2}$.

Cell treatment. Before the indicated treatments, cells were cultured in phenol red (PR)-free medium without serum for $24 \mathrm{~h}$. Cells were then treated with or without $10 \mathrm{nM}$ of E2 (or $1 \mu \mathrm{M} \mathrm{G1)}$ and different concentrations of baicalein $(5,10$, and $15 \mu \mathrm{M}$ ) in PR-free medium without serum for a specified period of time. Control cells were incubated in DMSO $(0.01 \%, \mathrm{v} / \mathrm{v})$.

MTT assay. The 96-well microculture plates were individually seeded with $100 \mu 1$ of the respective cell suspension and $100 \mu \mathrm{l}$ of treatment medium. After incubation at $37^{\circ} \mathrm{C}$ for $24 \mathrm{~h}, 20 \mu \mathrm{l}$ of MTT $(5 \mathrm{mg} / \mathrm{ml})$ was added, and the plates were incubated for an additional $4 \mathrm{~h}$ at $37^{\circ} \mathrm{C}$. The medium was then removed, and $100 \mu 1$ DMSO was added and mixed thoroughly. Spectrometric absorbance values at $570 \mathrm{~nm}$ were measured using a microplate reader (Bio-Rad, Lincoln, NE, USA). The results are expressed as inhibitory rates with respect to the controls, calculated using the following formula:

$$
\text { Inhibitory rate }(\%)=\left[\left(\mathrm{A}_{\text {control }}-\mathrm{A}_{\text {treated }}\right) / \mathrm{A}_{\text {control }}\right] \times 100 \text {. }
$$

Wound-healing assay. Cells were seeded into 12-well plates at $90 \%$ confluency. Monolayers were wounded with a micropipette tip and rinsed with phosphate-buffered saline (PBS) 3 times to remove any floating cells. Then the cells were incubated in the absence or presence of E2 $(10 \mathrm{nM})$ with or without various concentrations of baicalein $(5,10$, and $15 \mu \mathrm{M})$ for $24 \mathrm{~h}$. Cytosine $\beta$-D-arabinofuranoside hydrochloride $(10 \mu \mathrm{M})$ was added $1 \mathrm{~h}$ before the test treatment to inhibit cell proliferation (17). Images were captured at 0 and $24 \mathrm{~h}$ after wound application. The level of cell migration into the wound was quantified as the migration rate compared against the cells at $0 \mathrm{~h}$ for each group. 
A

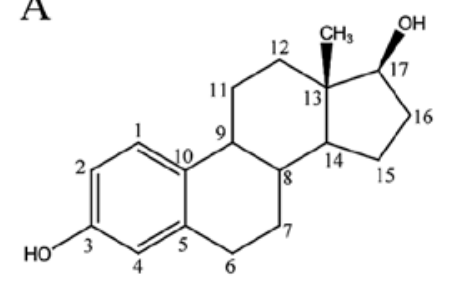

$17 \beta$-estradiol (E2)<smiles>Cc1c(O)c(O)c(O)c2c(=O)cc(-c3ccccc3)sc12</smiles>

Baicalein
C

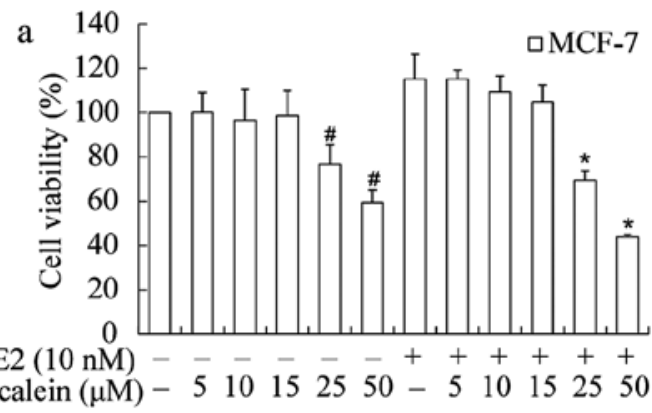
Baicalein $(\mu \mathrm{M})-510152550-510152550$
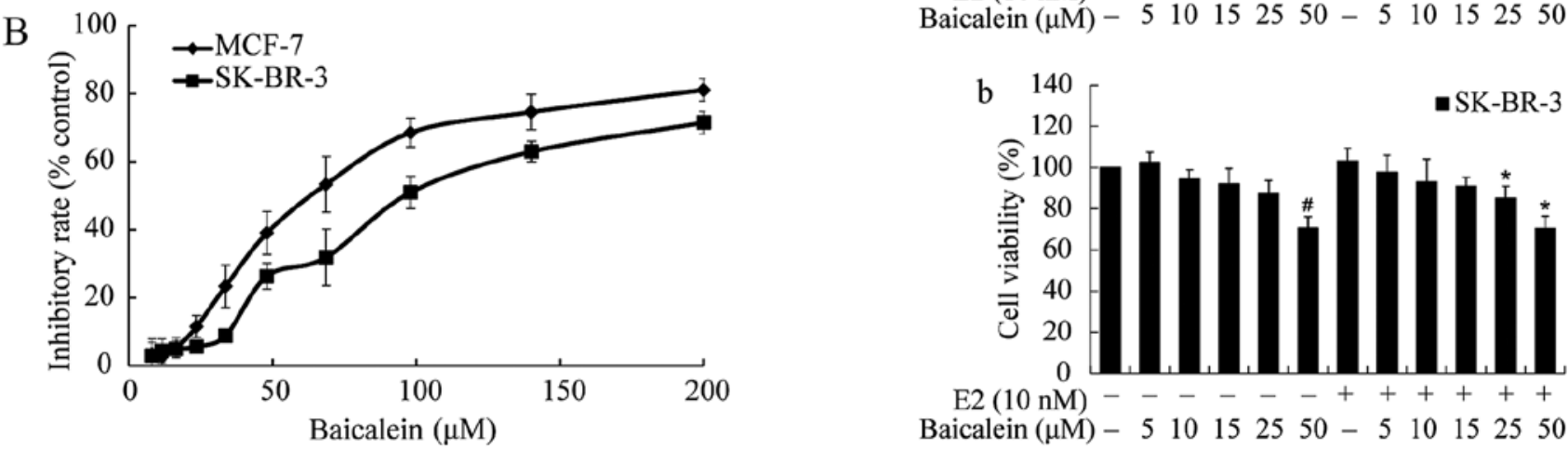

Figure 1. Effects of baicalein on cell viability. (A) Chemical structure of $17 \beta$-estradiol (E2) and baicalein. (B) Inhibitory effects of baicalein on the cell viability of MCF-7 and SK-BR-3 cells at $24 \mathrm{~h}$ as detected by MTT assay. Cells were treated with various concentrations of baicalein for $24 \mathrm{~h}$. The result is represented by the inhibitory rate. (C) Cells were incubated with the indicated doses of baicalein with or without E2 (10 nM) after $24 \mathrm{~h}$, and cellular viability was assessed in (a) MCF-7 and (b) SK-BR-3 cells. Values are mean \pm SEM. ${ }^{~} \mathrm{P}<0.05$ compared with control group, ${ }^{*} \mathrm{P}<0.05$ compared with the E2-treated group.

Cell adhesion assay. The cell adhesion assay was performed as described (18). Briefly, 96-well plates were coated with Matrigel $(50 \mu \mathrm{g} / \mathrm{well})$ at $37^{\circ} \mathrm{C}$ for $1 \mathrm{~h}$ and blocked with $0.5 \%$ BSA $(\mathrm{v} / \mathrm{v})$ at $4^{\circ} \mathrm{C}$ for $4 \mathrm{~h}$. After treatment with E2 $(10 \mathrm{nM})$ or E2 $(10 \mathrm{nM})$ plus baicalein $(5,10$, and $15 \mu \mathrm{M})$ for $24 \mathrm{~h}$, the cells were harvested and suspended in serum-free medium. Cells at a density of $2 \times 10^{5}$ cells $/ \mathrm{ml}(100 \mu \mathrm{l})$ were added into the plates and then incubated at $37^{\circ} \mathrm{C}$ for $1 \mathrm{~h}$. After incubating for adhesion, the medium was carefully suctioned out. The wells were then washed with PBS to remove non-adherent cells. The colorimetric MTT assay was employed to analyze the number of adherent cells. Spectrometric absorbance was measured at $570 \mathrm{~nm}$ by using a microplate reader (Bio-Rad). The results are expressed as adhesive cells with respect to the control as a percentage.

Invasion assay. Invasive ability of the cells was measured by assay using a Transwell chamber (Millipore, Billerica, MA, USA) containing membranes with an $8-\mu \mathrm{m}$ pore size and, coated with Matrigel as previously described (19). The cells were exposed to E2 (10 nM) with or without different concentrations of baicalein $(5,10$, and $15 \mu \mathrm{M})$ for $24 \mathrm{~h}$. The cells were then trypsinized and suspended at a final concentration of $5 \times 10^{5}$ cells $/ \mathrm{ml}$ in serum-free medium. The cell suspension was added into each 10-mm upper chamber, and medium with $10 \%$ FBS was added into the bottom chamber as a chemoattractant. After incubating for $24 \mathrm{~h}$, the upper surfaces of the membranes were swabbed to remove non-invaded cells, and the cells that attached to the lower surface were fixed in $100 \%$ methanol, stained with hematoxylin and eosin (H\&E) (Beyotime Institute of Biotechnology, Jiangsu, China), and counted under a microscope. Five randomly chosen fields were analyzed for each group.

Western blot analysis. Cells were collected after E2 (10 nM) or $\mathrm{G} 1(1 \mu \mathrm{M})$ with or without baicalein $(5,10$, and $15 \mu \mathrm{M})$ treatment for $1 \mathrm{~h}$, and lysed in lysis buffer (Beyotime Institute of Biotechnology). The lysates were clarified by centrifugation at $4^{\circ} \mathrm{C}$ for $15 \mathrm{~min}$ at $13,000 \mathrm{x} \mathrm{g}$. The concentration of protein in the supernatants was measured using bicinchoninic acid (BCA) assay kit (Beyotime Institute of Biotechnology) with a Varioskan Multimode microplate spectrophotometer (Thermo Waltham, MA, USA). Total proteins (30 $\mu \mathrm{g} /$ lane) were separated on a 12 or $8 \%$ SDS-PAGE gel, and transferred to a PVDF membrane (Millipore, Bedford, MA, USA). The membrane was blocked with 5\% non-fat milk and subsequently incubated with the indicated antibodies. Blots were visualized by using an enhanced chemiluminescence kit (Millipore, Billerica, MA, USA). Digital images of the blots were produced by a Syngene Gel Imaging System and quantified with GeneSnap (Syngene, Frederick, MD, USA) software.

Real-time reverse transcription-polymerase chain reaction $(R T-P C R)$. Total RNA was extracted after a 1-h treatment with $10 \mathrm{nM}$ E2 or G1 $(1 \mu \mathrm{M})$ in the presence or absence of baicalcein $(5,10$ and $15 \mu \mathrm{M})$ by using an RNA extraction kit (code no. 9767; Takara, Dalian, China) according to the manufacturer's instructions. Complementary DNA (cDNA) was synthesized using a Strand Complementary DNA Synthesis kit (code no.6210A; Takara). Real time PCR was performed on an iCycle iQ (Bio-Rad) using $20 \mathrm{ng}$ of cDNA template and $10 \mu \mathrm{M}$ primers. Cysteine-rich 61 (CYR61) primers (forward, 5'-ACT 
A
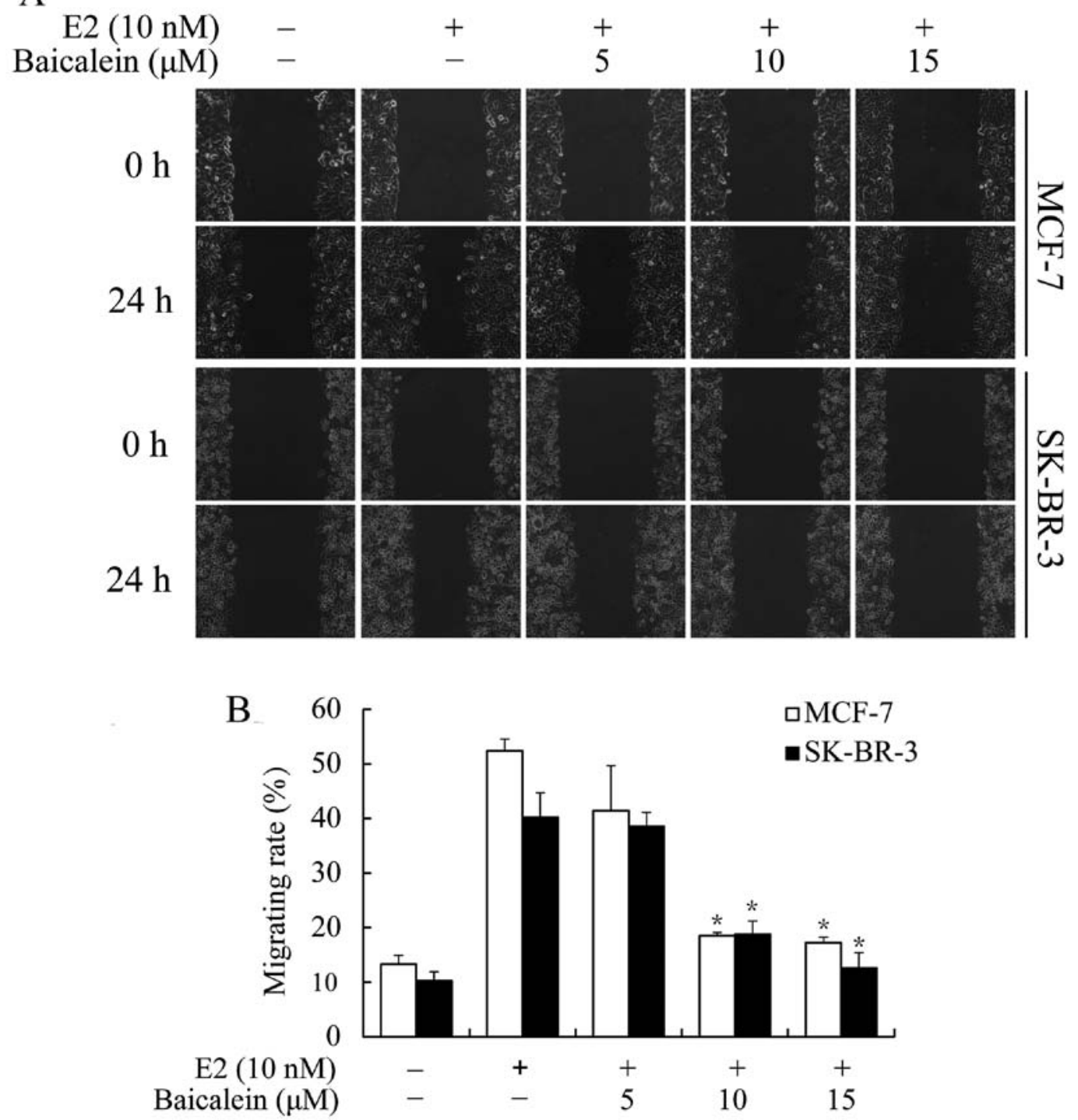

Figure 2. Effects of baicalein on the E2-induced migration of MCF-7 and SK-BR-3 breast cancer cells. (A) Confluent monolayers were scratched and incubated in the absence or presence of E2 $(10 \mathrm{nM})$ with or without various concentrations of baicalein $(5,10$, and $15 \mu \mathrm{M})$ for $24 \mathrm{~h}$. The images were captured at 0 and $24 \mathrm{~h}$ after wounding (magnification, x100). These figures are representative of 3 separate experiments. (B) The inhibition of E2-stimulated migration by baicalein. Values are mean \pm SEM. ${ }^{*} \mathrm{P}<0.05$ compared with the E2-treated group. E2, 17 $\beta$-estradiol.

TCA TGG TCC CAG TGC TC-3' and reverse, 5'-AAA TCC GGG TTT CTT TCA CA-3') (20), connective tissue growth factor $(C T G F)$ primers (forward, 5'-ACC TGT GGG ATG GGC ATC T-3' and reverse, 5'-CAG GCG GCT CTG CTT CTC TA-3') (21) and $\beta$-actin primers (forward, 5'-AGT TGC GTT ACA CCC TTT C-3' and reverse, 5'-CCT TCA CCG TTC CAG TTT-3') (22) were synthesized by Sangon Biotech (Shanghai, China). The mRNA levels were measured using SYBR Premix Ex Taq ${ }^{\mathrm{TM}}$ (code no. RR037A; Takara). The reactions were performed for $30 \mathrm{sec}$ at $95^{\circ} \mathrm{C}$, followed by 40 cycles at $95^{\circ} \mathrm{C}$ for $5 \mathrm{sec}$ and $60^{\circ} \mathrm{C}$ for $30 \mathrm{sec}$. The melting curve was analyzed at 55 to $95^{\circ} \mathrm{C}$ to detect a single genespecific peak and to verify the absence of primer dimer peaks. The $C Y R 61$ or $C T G F$ mRNA level was quantified in relation to $\beta$-actin mRNA level by using the $2^{-\Delta \Delta C t}$ method.

Statistical analysis. Statistically significant differences were calculated by one-way ANOVA followed by the Bonferroni post-hoc test for multiple-group comparisons. A $\mathrm{P}<0.05$ was considered to indicate a significant difference, and data are expressed as mean \pm standard error of the mean (SEM). The data shown in this test were obtained from 3 independent experiments.

\section{Results}

Cytotoxic effect of baicalein on MCF-7 and SK-BR-3 cells. To investigate the effect of baicalein on cell viability, MCF-7 and SK-BR-3 cells were treated with various concentrations of baicalein for $24 \mathrm{~h}$, and viability was detected by MTT assay. The $\mathrm{IC}_{50}$ values of baicalein in the MCF-7 and SK-BR-3 cells after $24 \mathrm{~h}$ were $66.3 \pm 5.9$ and $103.5 \pm 9.4 \mu \mathrm{M}$, respectively (Fig. 1B). According to the results, baicalein at 5, 10 and $15 \mu \mathrm{M}$ had no obvious cytotoxic effect on the MCF-7 or SK-BR-3 cells in the presence or absence of E2 (Fig. 1C), and were used in following study. 

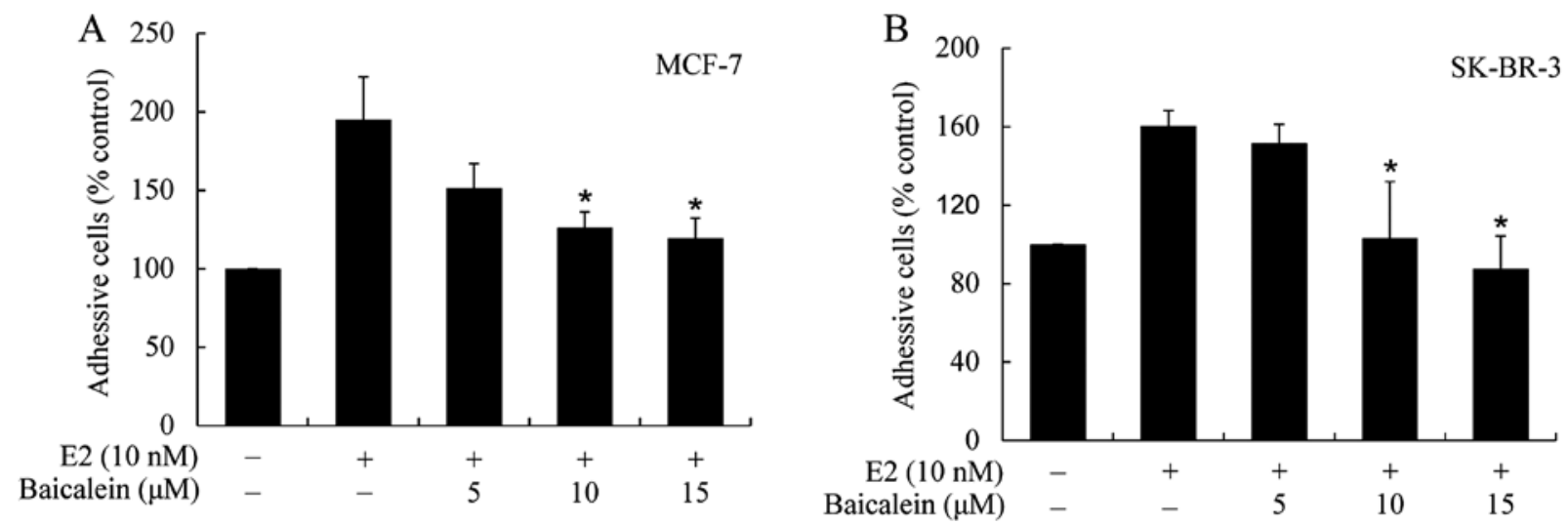

Figure 3. Effects of baicalein on E2-promoted cell adhesion to Matrigel in (A) MCF-7 and (B) SK-BR-3 cells. Cells were treated with or without E2 (10 nM) or E2 $(10 \mathrm{nM})$ plus baicalein $(5,10$ and $15 \mu \mathrm{M})$ for $24 \mathrm{~h}$. The results are expressed as adhesive cells with respect to the control (as $100 \%)$. ${ }^{*} \mathrm{P}<0.05$ indicates significant difference from the E2-treated group. Values are mean \pm SEM. E2, 17 $\beta$-estradiol.
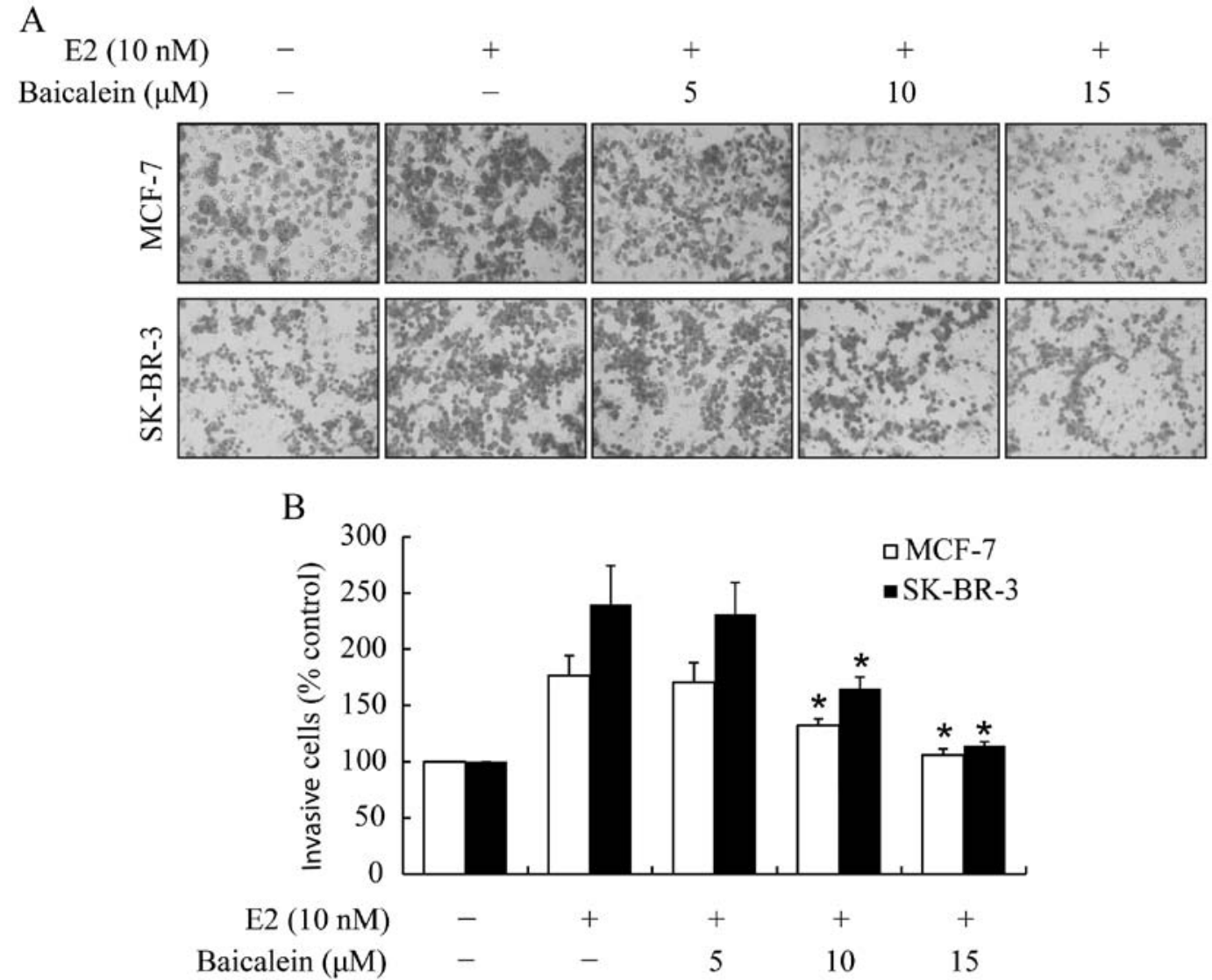

Figure 4. Effect of baicalein on E2-stimulated cell invasion in MCF-7 and SK-BR-3 cells. (A) Cells were treated with or without E2 (10 nM) and different concentrations of baicalein $(5,10$ and $15 \mu \mathrm{M})$ for $24 \mathrm{~h}$. Invasive cells that passed through the membrane were evaluated using H\&E staining (magnification, $\mathrm{x} 200$ ). The images are representative of 3 separate experiments. (B) The results are expressed as invasive cells with respect to the control (as $100 \%)$. Values are mean \pm SEM. ${ }^{*} \mathrm{P}<0.05$ indicates significant difference from the E2-treated group. E2, 17ß-estradiol.

Baicalein inhibits E2-induced cell migration. After treatment with E2, MCF-7 and SK-BR-3 cells were found to have significantly enhanced migration into the wound, while cells treated with baicalein had less migratory ability (Fig. 2A). E2 stimulation for $24 \mathrm{~h}$ significantly increased the migration rate from $13 \pm 2$ to $52 \pm 3 \%$ in the MCF-7 cells, and from $10 \pm 4$ to $40 \pm 5 \%$ in the SK-BR-3 cells, respectively. After treatment with $15 \mu \mathrm{M}$ baicalein for $24 \mathrm{~h}$, the migration rate decreased to $17 \pm 2 \%$ in the MCF-7 cells and $13 \pm 4 \%$ in the SK-BR-3 cells, respectively (Fig. 2B). These results demonstrated that baicalein suppressed E2-induced cell migration.

Baicalein suppresses E2-promoted cell adhesion to Matrigel. Adhesion of tumor cells to extracellular matrix (ECM) is an essential step for tumor invasion and metastasis. We next examined the effect of baicalein on E2-promoted cell adhesion 

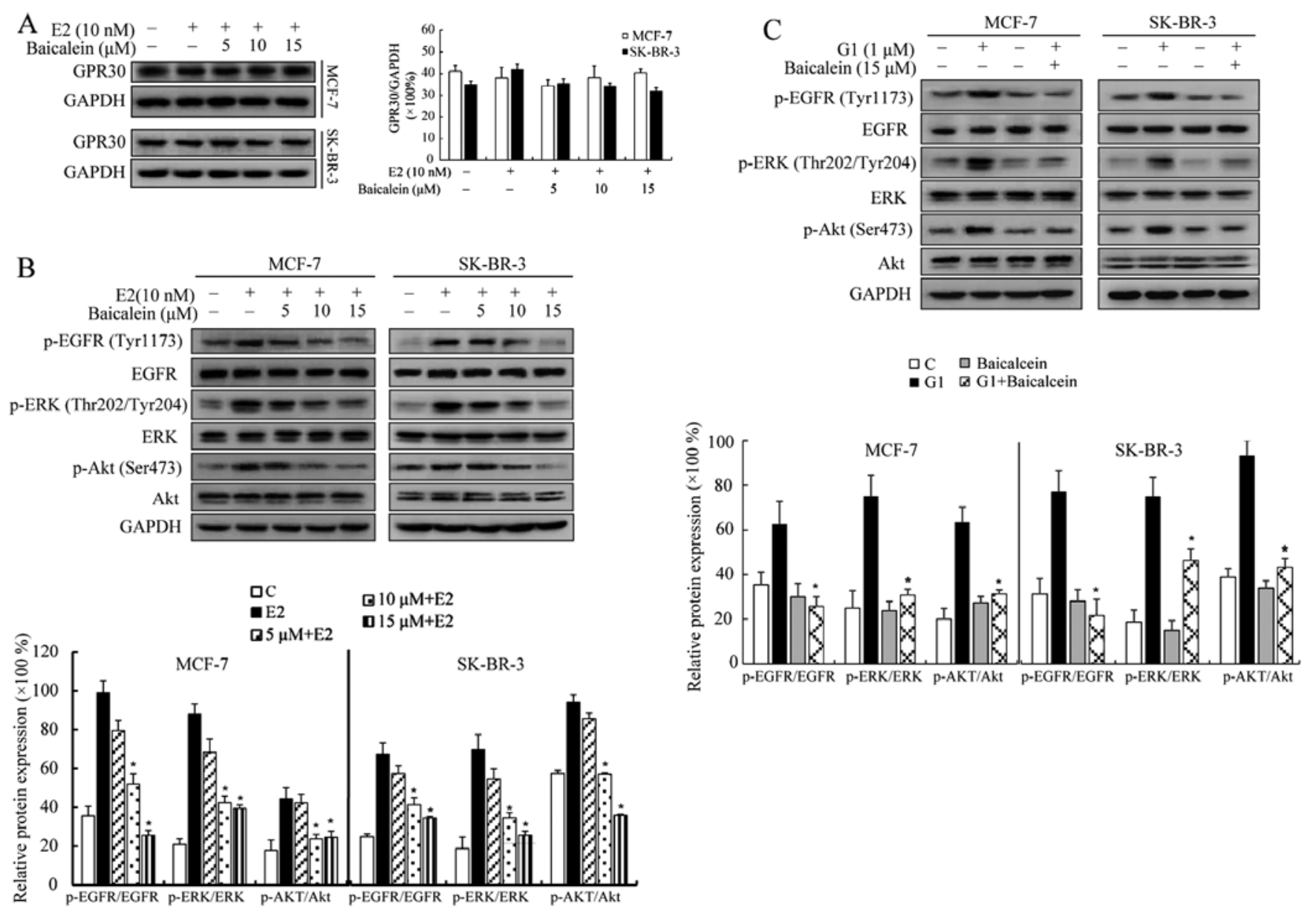

Figure 5. Effect of baicalein on E2-stimulated activation of the GPR30 signaling pathway. The cells were harvested after E2 (10 nM) with or without baicalein $(5,10$ and $15 \mu \mathrm{M})$ treatment for $1 \mathrm{~h}$. (A) Western blot assay was used to examine the expression of GPR30. GAPDH was used as a loading control. (B) The total and phosphorylated protein levels of EGFR, ERK1/2 and Akt. "P<0.05, significantly different from the E2-treated group. (C) The total and phosphorylated protein levels of EGFR, ERK1/2 and Akt in the cells treated with G1 $(1 \mu \mathrm{M})$ in the presence or absence of baicalein $(15 \mu \mathrm{M})$ treatment for $1 \mathrm{~h}$. "P<0.05 vs. the G1-treated group. Densitometric analysis of the relative ratios of GPR30/GAPDH, phosphorylated EGFR (Try 1173)/total EGFR, phosphorylated ERK1/2 (Thr 202/Tyr 204)/total ERK1/2 and phosphorylated Akt (Ser473)/total Akt. Values are mean \pm SEM. E2, 17ß-estradiol; GPR30, G protein-coupled estrogen receptor; ERK, extracellular signal-regulated kinase; EGFR, epidermal growth factor receptor.

to Matrigel, a reconstituted basement membrane (an important component of ECM). E2 greatly increased cell-Matrigel adhesion by $195.0 \pm 27 \%$ in the MCF-7 and by $161 \pm 8 \%$ in the SK-BR-3 cells, when compared with the control (Fig. 3). Treatment with 5,10 , and $15 \mu \mathrm{M}$ baicalein reduced the adhesive cells to $151 \pm 16,126 \pm 10$, and $119 \pm 13 \%$ in the MCF-7 cells (Fig. 3A); and $152 \pm 5,103 \pm 15$, and $89 \pm 9 \%$ in the SK-BR-3 cells (Fig. 3B), respectively. The data showed that baicalein effectively suppressed E2-promoted cell adhesion to Matrigel.

Baicalein reduces E2-stimulated cell invasion. The Matrigel-coated Transwell chamber system was used to investigate the effects of baicalein on E2-enhanced cell invasion. After incubation with E2 for $24 \mathrm{~h}$, the invaded cells across the Matrigel-coated membrane were increased in both cell lines (Fig. 4A). In the MCF-7 cell line, the invaded cells were increased to $176 \pm 18 \%$ in the E2 treatment group compared with the control, but decreased to $132 \pm 6$ and $106 \pm 5 \%$ in the 10 and $15 \mu \mathrm{M}$ baicalein treatment groups, respectively (Fig. 4B). Similar results were observed in the SK-BR-3 cells. After SK-BR-3 cells were treated with baicalein, the invaded cells in the E2-treated group decreased from $240 \pm 34$ to $231 \pm 28 \%, 165 \pm 10 \%$ and $114 \pm 4 \%$ in the 5,10 and $15 \mu \mathrm{M}$ treatment groups, respectively (Fig. 4B). Baicalein was evidently able to suppress E2-enhanced cell invasion.

Baicalein prevents E2-induced GPR30 signal transduction. The transmembrane receptor, GPR30, is able to mediate estrogen responsiveness in ER-positive or ER-negative breast cancer cells. Here, we aimed to determine whether or not baicalein has inhibitory effects on GPR30 expression or its signal transduction. We observed that GPR30 expression remained unchanged after exposure to E2 with or without baicalein in both the MCF-7 and SK-BR-3 cells (Fig. 5A). Previous studies have indicated that GPR 30 activation triggers EGFR transactivation by tyrosine phosphorylation in breast cancer cells $(23,24)$, thus we sought to evaluate the influence of baicalein in EGFR phosphorylation upon E2 stimulation. When cells were treated with baicalein in the presence of E2, there was a significant decrease in EGFR phosphorylation (Tyr 1173) at 10 and $15 \mu \mathrm{M}$ (Fig. 5B). GPR30 triggers EGFR transactivation leading to the phosphorylation of ERK1/2 (24) 

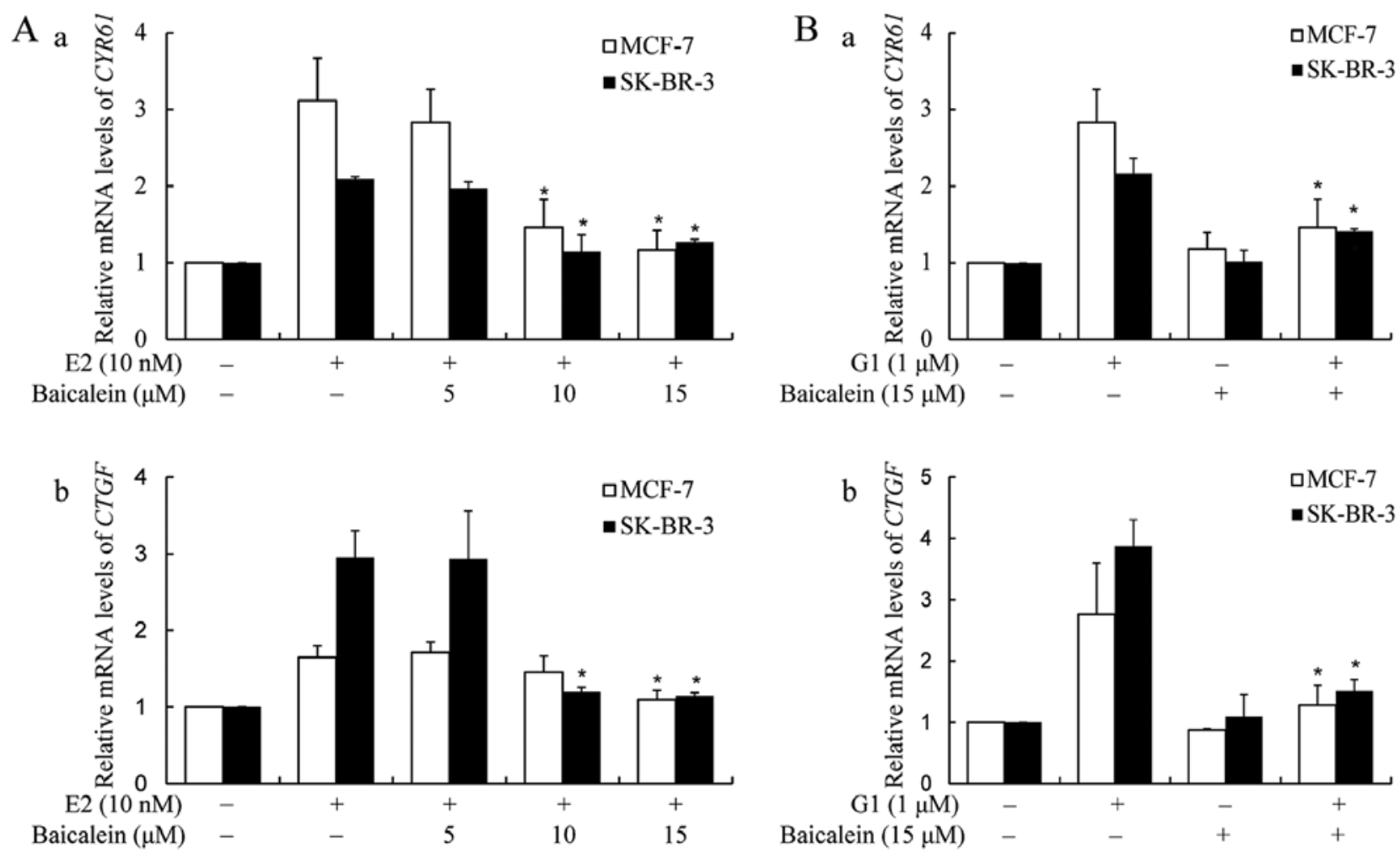

Figure 6. Effect of baicalein on E2-induced GPR30 target gene transcription. The CYR61 and CTGF mRNA expression levels were detected by real-time PCR. (A) The cells were treated with $10 \mathrm{nM} \mathrm{E2}$ in the presence or absence of baicalcein $(5,10$ and $15 \mu \mathrm{M})$ for $1 \mathrm{~h}$. Relative mRNA levels of (a) $C Y R 61$ and (b) $C T G F .{ }^{*} \mathrm{P}<0.05$ vs. E2-treated group. (B) The cells were treated with $1 \mu \mathrm{M} \mathrm{G} 1$ with or without $15 \mu \mathrm{M}$ baicalcein for $1 \mathrm{~h}$. Relative mRNA levels of (a) $C Y R 61$ and (b) $C T G F$. "P<0.05 vs. the G1-treated group. Results obtained from experiments performed in triplicate were normalized to the $\beta$-actin mRNA level and are shown as fold changes compared to the control cells. Values are mean \pm SEM. E2, 17ß-estradiol; GPR30, G protein-coupled estrogen receptor; CYR61, cysteine-rich $61 ; C T G F$, connective tissue growth factor.

and activation of the PI3K/Akt transduction pathway (25). Thus, it was essential to analyze the phosphorylation status of ERK1/2 and Akt. Consequently, E2-induced phosphorylation of ERK1/2 at Thr 202/Tyr 204 and Akt at Ser 373 were repressed in the presence of baicalein at 10 and $15 \mu \mathrm{M}$ (Fig. 5B). In addition, GPR30 agonist G1 was used to further confirm whether baicalein interferes with GPR30 signal transduction. Indeed, treatment with baicalein at $15 \mu \mathrm{M}$ prevented G1-induced EGFR, ERK and Akt phosphorylation (Fig. 5C).

Baicalein suppresses E2-induced GPR30-mediated gene expression. Previous reports have shown that $C Y R 61$ and $C T G F$ are the target genes which mediate the stimulatory effects triggered by GPR30 activation (26). The potential of baicalein to regulate GPR30-mediated expression of both target genes was detected by real-time PCR. E2 upregulated CYR61 and $C T G F$ mRNA levels in the 2 cell lines. The downregulation of the 2 genes in the MCF-7 and SK-BR-3 cells (Fig. 6A) was observed after exposure to baicalein, when compared with the E2-treated groups. In addition, the upregulation of CYR61 and $C T G F$ mRNA levels induced by $\mathrm{G} 1$ in the MCF-7 and SK-BR-3 cells was also inhibited in the presence of baicalein at $15 \mu \mathrm{M}$ (Fig. 6B).

\section{Discussion}

Estrogens are essential for the growth and development of mammary glands (27). However, prolonged exposure to endogenous or exogenous estrogens is a major risk factor for the development of breast cancer (28). Estrogens have been confirmed to play a key role in breast cancer metastasis $(29,30)$. Tumor metastasis consists of a series of highly relevant biological processes including disruption of cell-cell and augmentation of cell-ECM adhesion capacity, induction of cellular motility, invasiveness to the basement membrane and ECM constituents, angiogenesis, and formation of a metastatic lesion (31). In the present study, baicalein treatment inhibited cell migration in response to E2 stimulation. Furthermore, it suppressed E2-enhanced cell adhesion to Matrigel. Moreover, it significantly decreased E2-promoted invasive ability across a Transwell membrane coated with Matrigel. These results imply that baicalein may possess anti-estrogenic ability to suppress E2-induced migration, adhesion and invasion of MCF-7 and SK-BR-3 cells in vitro.

GPR30 is independent of the ER status in breast cancer cells and tissue samples, and its action differs from the classical nuclear ERs, ER $\alpha$ and ER $\beta$ (7). Recent studies have demonstrated that GPR30 mediates E2-enhanced cell migration and invasion in triple-negative or ER $\alpha$-negative breast cancer cells $(32,33)$. Furthermore, we recently found that GPR30 mediates E2-induced calpain activation, which is correlated with the metastatic phenotype of breast cancer, in both ER $\alpha$-positive and -negative cells (34). We also showed that tamoxifen, an ER antagonist but a GPR30 agonist, could not suppress E2-promoted migration but enhanced migration in breast cancer cells and induced truncation of cyclin $\mathrm{E}$ 
via GPR30 (35). It appears that GPR30, regardless of ER $\alpha$ expression, plays an important role in E2-enhanced migration and invasion of breast cancer cells. Our results showed that baicalein suppressed E2-promoted migration and invasion in GPR30-positive/ER $\alpha$-negative SK-BR-3 cells and also in GPR30/ER $\alpha$-positive MCF-7 cells. Although baicalein had little effect on GRP30 expression, it markedly inhibited the E2-induced GPR30 signal activation, by decreasing EGFR phosphorylation and activation of ERK1/2 and Akt. Meanwhile, baicalein suppressed G1-induced upregulation of the phosphorylation of EGFR, ERK1/2 and Akt. The results indicate that the inhibitive effect of baicalein on the response of MCF-7 and SK-BR-3 cells to estrogen may derive partly from interfering with GPR30 signal transduction.

CYR61 and CTGF, also known as CCN1 and CCN2, belong to the $\mathrm{CCN}$ protein family, which exerts their biological functions by binding and activating cell surface integrins to stimulate cell growth, adhesion, matrix production and migration, and by regulating angiogenesis and tumorigenesis (36). CYR61 and CTGF expression is significantly associated with tumor size and lymph node metastasis, suggesting that they play a role in the progression of breast cancer (20). CYR61 is a survival and proangiogenic factor and induces metastasis and drug resistance in breast cancer cells (37). CTGF enhances migratory/invasive processes in human breast cancer by activating the integrin$\alpha v \beta 3$-ERK1/2-dependent upregulation of S100 calcium-binding protein A4 (S100A4) (38). The two genes related to breast cancer metastasis are the target genes of GPR30. Here, we found that baicalein significantly inhibited E2- or G1-induced upregulation of CTGF and CYR61 gene expression. These results suggest that baicalein may prevent E2-induced migration, invasion and adhesion of MCF-7 and SK-BR-3 cells also by suppressing GPR30-dependent gene transcription.

Flavonoids possess a chemical structure similar to estrogen, and some have been considered to exert an anti-estrogenic effect and reduce the risk of breast cancer. Genistein, which is the main ingredient responsible for the cancer chemopreventive activity of soy-derived foods, has garnered much research. However, it displays a biphasic effect on the proliferation and death of MCF-7 cells and displays estrogenic activity to transactivate $\mathrm{ER} \alpha$ at low concentrations. Although baicalein is an isomer of genistein, it does not have a biphasic effect on ER, which suggests that it could be a better chemopreventive agent than genistein (14). Moreover, genistein can stimulate GPR30 to mediate c-fos upregulation in breast cancer cells (39) and induce the proliferation of thyroid cancer cells (40). In the present study, we found that baicalein suppressed E2 or G1-induced GPR30 signal activation indicating a different activity from genistein. The precise difference between the two compounds with similar chemical structure remains to be resolved in future studies. The relationship between the structure of flavonoids and the inhibition of E2 events warrants further study. To fully investigate the mechanism, we need to explain the detailed molecular mechanisms with which baicalein influences GPR30 and downstream signaling, as well as crosstalks with other receptors. In addition, whether baicalein has dual effects on both ER $\alpha$ and GPR30 still needs further investigation.

In conclusion, baicalein attenuates E2-induced migration, adhesion and invasion of MCF-7 and SK-BR-3 breast cancer cells through interference with GPR30 pathway activation. This indicates that baicalein may be a promising agent for the treatment of GPR30-positive breast cancer metastasis.

\section{Acknowledgements}

The present study was supported by the Natural Science Foundation of Guizhou Province of China [grant no. QKHJ(2014)2007], the National Natural Science Foundation of China (grant nos. 81302804 and 31360252), Startup Foundation for Doctors of Guiyang Medical University [grant no. (2013)09] and the Foundation for Training Programs of Innovation and Entrepreneurship for Undergraduates of Guiyang Medical University (grant no. 201410660038).

\section{References}

1. Jemal A, Bray F, Center MM, Ferlay J, Ward E and Forman D: Global cancer statistics. CA Cancer J Clin 61: 69-90, 2011.

2. Libson $\mathrm{S}$ and Lippman M: A review of clinical aspects of breast cancer. Int Rev Psychiatry 26: 4-15, 2014.

3. Yager JD and Davidson NE: Estrogen carcinogenesis in breast cancer. N Engl J Med 354: 270-282, 2006.

4. Planey SL, Kumar R and Arnott JA: Estrogen receptors (ERo versus ER $\beta$ ): friends or foes in human biology. J Recept Signal Transduct Res 34: 1-5, 2014.

5. Herynk MH and Fuqua SA: Estrogen receptors in resistance to hormone therapy. Adv Exp Med Biol 608: 130-143, 2007.

6. Prossnitz ER and Maggiolini M: Mechanisms of estrogen signaling and gene expression via GPR30. Mol Cell Endocrinol 308: 32-38, 2009.

7. Maggiolini M and Picard D: The unfolding stories of GPR30, a new membrane-bound estrogen receptor. J Endocrinol 204: 105-114, 2010.

8. Filardo EJ, Graeber CT, Quinn JA, Resnick MB, Giri D, DeLellis RA, Steinhoff MM and Sabo E: Distribution of GPR30, a seven membrane-spanning estrogen receptor, in primary breast cancer and its association with clinicopathologic determinants of tumor progression. Clin Cancer Res 12: 6359-6366, 2006.

9. Berger CE, Qian Y, Liu G, Chen H and Chen X: p53, a target of estrogen receptor (ER) $\alpha$, modulates DNA damage-induced growth suppression in ER-positive breast cancer cells. J Biol Chem 287: 30117-30127, 2012.

10. Liu Q, Li JG, Zheng XY, Jin F and Dong HT: Expression of CD133, PAX2, ESA, and GPR30 in invasive ductal breast carcinomas. Chin Med J (Engl) 122: 2763-2769, 2009.

11. Takemura H, Sakakibara H, Yamazaki S and Shimoi K: Breast cancer and flavonoids - a role in prevention. Curr Pharm Des 19: 6125-6132, 2013.

12. Rosenberg Zand RS, Jenkins DJ and Diamandis EP: Flavonoids and steroid hormone-dependent cancers. J Chromatogr B Analyt Technol Biomed Life Sci 777: 219-232, 2002.

13. Srinivas NR: Baicalin, an emerging multi-therapeutic agent: pharmacodynamics, pharmacokinetics, and considerations from drug development perspectives. Xenobiotica 40: 357-367, 2010.

14. Po LS, Chen ZY, Tsang DS and Leung LK: Baicalein and genistein display differential actions on estrogen receptor (ER) transactivation and apoptosis in MCF-7 cells. Cancer Lett 187: 33-40, 2002.

15. Shenouda NS, Zhou C, Browning JD, Ansell PJ, Sakla MS, Lubahn DB and Macdonald RS: Phytoestrogens in common herbs regulate prostate cancer cell growth in vitro. Nutr Cancer 49: 200-208, 2004.

16. Lin CW, Yang LY, Shen SC and Chen YC: IGF-I plus E2 induces proliferation via activation of ROS-dependent ERKs and JNKs in human breast carcinoma cells. J Cell Physiol 212: 666-674, 2007.

17. Li Y, Wang JP, Santen RJ, Kim TH, Park H, Fan P and Yue W: Estrogen stimulation of cell migration involves multiple signaling pathway interactions. Endocrinology 151: 5146-5156, 2010.

18. Qi Q, Lu N, Wang XT, Gu HY, Yang Y, Liu W, Li C, You QD and Guo QL: Anti-invasive effect of gambogic acid in MDA-MB-231 human breast carcinoma cells. Biochem Cell Biol 86: 386-395, 2008. 
19. Chen P, Lu N, Ling Y, Chen Y, Hui H, Lu Z, Song X, Li Z, You Q and Guo Q: Inhibitory effects of wogonin on the invasion of human breast carcinoma cells by downregulating the expression and activity of matrix metalloproteinase-9. Toxicology 282: $122-128,2011$

20. Xie D, Nakachi K, Wang H, Elashoff R and Koeffler HP: Elevated levels of connective tissue growth factor, WISP-1, and CYR61 in primary breast cancers associated with more advanced features. Cancer Res 61: 8917-8923, 2001.

21. Pupo M, Pisano A, Lappano R, Santolla MF, De Francesco EM, Abonante S, Rosano C and Maggiolini M: Bisphenol A induces gene expression changes and proliferative effects through GPER in breast cancer cells and cancer-associated fibroblasts. Environ Health Perspect 120: 1177-1182, 2012.

22. Ferreira E and Cronjé MJ: Selection of suitable reference genes for quantitative real-time PCR in apoptosis-induced MCF-7 breast cancer cells. Mol Biotechnol 50: 121-128, 2012.

23. Filardo EJ, Quinn JA and Sabo E: Association of the membrane estrogen receptor, GPR30, with breast tumor metastasis and transactivation of the epidermal growth factor receptor. Steroids 73: 870-873, 2008

24. Filardo EJ, Quinn JA, Bland KI and Frackelton AR Jr: Estrogen-induced activation of Erk-1 and Erk-2 requires the G protein-coupled receptor homolog, GPR30, and occurs via trans-activation of the epidermal growth factor receptor through release of HB-EGF. Mol Endocrinol 14: 1649-1660, 2000

25. Ge C, Yu M and Zhang C: G protein-coupled receptor 30 mediates estrogen-induced proliferation of primordial germ cells via EGFR/Akt/ $\beta$-catenin signaling pathway. Endocrinology 153 : 3504-3516, 2012

26. Pandey DP, Lappano R, Albanito L, Madeo A, Maggiolini M and Picard D: Estrogenic GPR30 signalling induces proliferation and migration of breast cancer cells through CTGF. EMBO J 28 : 523-532, 2009

27. Stingl J: Estrogen and progesterone in normal mammary gland development and in cancer. Horm Cancer 2: 85-90, 2011.

28. Haldosén LA, Zhao C and Dahlman-Wright K: Estrogen receptor beta in breast cancer. Mol Cell Endocrinol 382: 665-672, 2014

29. Ganapathy V, Banach-Petrosky W, Xie W, Kareddula A, Nienhuis H,Miles G and Reiss M: Luminal breast cancer metastasis is dependent on estrogen signaling. Clin Exp Metastasis 29: 493-509, 2012.

30. Zheng S, Huang J, Zhou K, Zhang C, Xiang Q, Tan Z, Wang T and $\mathrm{Fu} X: 17 \beta$-estradiol enhances breast cancer cell motility and invasion via extra-nuclear activation of actin-binding protein ezrin. PLoS One 6: e22439, 2011.
31. Perlikos F, Harrington KJ and Syrigos KN: Key molecular mechanisms in lung cancer invasion and metastasis: a comprehensive review. Crit Rev Oncol Hematol 87: 1-11, 2013.

32. Yu T, Liu M, Luo H, Wu C, Tang X, Tang S, Hu P, Yan Y, Wang $\mathrm{Z}$ and Tu G: GPER mediates enhanced cell viability and motility via non-genomic signaling induced by $17 \beta$-estradiol in triple-negative breast cancer cells. J Steroid Biochem Mol Biol 143: 392-403, 2014.

33. Jiang QF, Wu TT, Yang JY, Dong CR, Wang N, Liu XH and Liu ZM: $17 \beta$-estradiol promotes the invasion and migration of nuclear estrogen receptor-negative breast cancer cells through cross-talk between GPER1 and CXCR1. J Steroid Biochem Mol Biol 138: 314-324, 2013.

34. Chen Y, Li Z, He Y, Shang D, Pan J, Wang H, Chen H, Zhu Z, Wan L and Wang X: Estrogen and pure antiestrogen fulvestrant (ICI 182 780) augment cell-matrigel adhesion of MCF-7 breast cancer cells through a novel $\mathrm{G}$ protein coupled estrogen receptor (GPR30)-to-calpain signaling axis. Toxicol Appl Pharmacol 275: 176-181, 2014.

35. Li Y, Chen Y, Zhu ZX, Liu XH, Yang L, Wan L, Lei TW and Wang XD: 4-Hydroxytamoxifen-stimulated processing of cyclin $\mathrm{E}$ is mediated via $\mathrm{G}$ protein-coupled receptor 30 (GPR30) and accompanied by enhanced migration in MCF-7 breast cancer cells. Toxicology 309: 61-65, 2013.

36. Jun JI and Lau LF: Taking aim at the extracellular matrix: $\mathrm{CCN}$ proteins as emerging therapeutic targets. Nat Rev Drug Discov 10: 945-963, 2011.

37. Espinoza I, Liu H, Busby R and Lupu R: CCN1, a candidate target for zoledronic acid treatment in breast cancer. Mol Cancer Ther 10: 732-741, 2011.

38. Chen PS, Wang MY, Wu SN, et al: CTGF enhances the motility of breastcancercells viaanintegrin-alphavbeta3-ERK1/2-dependent S100A4-upregulated pathway. J Cell Sci 120: 2053-2065, 2007.

39. Maggiolini M, Vivacqua A, Fasanella G, Recchia AG, Sisci D, Pezzi V, Montanaro D, Musti AM, Picard D and Andò S: The G protein-coupled receptor GPR30 mediates c-fos up-regulation by 17 beta-estradiol and phytoestrogens in breast cancer cells. J Biol Chem 279: 27008-27016, 2004.

40. Vivacqua A, Bonofiglio D, Albanito L, Madeo A, Rago V, Carpino A, Musti AM, Picard D, Andò S and Maggiolini M: 17beta-estradiol, genistein, and 4-hydroxytamoxifen induce the proliferation of thyroid cancer cells through the g protein-coupled receptor GPR30. Mol Pharmacol 70: 1414-1423, 2006. 